\title{
Review of Dynamic-Force Correction Method for a Force Transducer with the Levitation Mass Method
}

\author{
Akihiro Takita $^{\mathrm{a},{ }^{*}}$, Yoshiki Ono ${ }^{\mathrm{a}}$, Yusaku Fujii ${ }^{\mathrm{a}}$ \\ ${ }^{a}$ Department of Electronic Engineering, Faculty of Engineering, Gunma University, \\ 1-5-1 Tenjin-cho, Kiryu, Gunma 376-8515, Japan \\ *Corresponding Author: takita@el.gunma-u.ac.jp
}

\begin{abstract}
In this paper, the measurement and correction method of the dynamic error of a force transducer are reviewed. The dynamic force, an impact load in this paper, is applied to an S-shaped strain-gauge force transducer is measured with using the Levitation Mass Method (LMM). In the LMM, the force acting on the force transducer is measured as a reaction force acting on a moving part of an aerostatic linear bearing which is collided with the force transducer. The force acting on the moving part is measured as an inertial force of the moving part. Finally, the inertial force is calculated by the mass and the acceleration of the moving part. As a result of comparing the forces measured by the transducer and by the LMM, it is shown that the dynamic error can be estimated by the second time derivative of the output of the transducer. Therefore, the dynamic error of the force transducer can be corrected by using the output data from the transducer itself, without using the LMM.
\end{abstract}

Keywords: force transducer, dynamic force measurement, levitation mass method.

\section{Introduction}

Force transducers are widely used in industrial and research areas. For static force calibration for the transducers, some methods that use the static standard force are established. However, there are no standard methods for dynamic force calibration. This shows that it is very difficult to estimate the dynamic error of force transducers and correct the error.

Some researchers have proposed methods for evaluating the dynamic error of force transducers. Kumme proposed a method in which an oscillation force is applied to a force transducer. ${ }^{(1,2)}$ In this method, a mass is attached to the sensing part of the transducer, and the transducer with the mass is vertically attached to a shaker. The oscillation force applied to the transducer is the inertial force of the mass. our laboratory proposed a method, the levitation mass method (LMM), in which varying forces such as an impact force, an oscillation force, and a step force are applied to the transducer as the inertial force of a mass supported using an aerostatic linear bearing. ${ }^{(3-4)}$ In this method, only the Doppler frequency shift of the laser light reflected on the mass is accurately measured, using an optical interferometer. All the other quantities such as velocity, acceleration, displacement, and inertial force are then calculated from the frequency obtained. Based on the dynamic force measurement, we developed the correction method for force transducer on dynamic condition with and without using the LMM. ${ }^{(5-7)}$

In this paper, we review the method for measuring dynamic force applied to a force transducer and the method for correcting the difference between the applied force and the force measured by the transducer.

\section{Experimental Setup}

Figure 1 shows a schematic diagram of the experimental setup for measuring the dynamic response of the force transducers. The transducer being tested is fixed on a base. An aerostatic linear bearing is used to achieve linear motion with sufficiently small friction acting on the mass, i.e., the moving part of the bearing. ${ }^{(8)}$ The moving part with a corner cube prism (CC) and an extension rod is given its initial velocity by hand, and the moving part then collides with the sensing point of the force transducer.

A rubber block of sufficiently small mass is attached to 
the sensing point to adjust the steepness of the impact. The velocity of the mass $v_{1}$ and the velocity of the sensing point of the transducer $v_{2}$ are measured using two optical interferometers, called interferometer1 and interferometer2, respectively. The force acting on the sensing point is calculated as reaction force acting on the moving part by the equation of motion, $F=m a$, where $m$ is the mass and $a$ is the acceleration of the moving part.

The mass of the moving part, including the $\mathrm{CC}$ and the extension rod, M1, is $2.653 \mathrm{~kg}$. The mass of the contact point of the transducer, including the $\mathrm{CC}$ and their base plate, $\mathrm{M} 2$, is $0.082 \mathrm{~kg}$.

A conventional S-shaped strain-gage type transducer (model: DB-200N, manufacturer: Showa Measuring Instruments Co., Ltd., capacity: $200 \mathrm{~N}$ ), was used in the experiment.

A Zeeman-type two-frequency He-Ne laser was used as the light source of the interferometers. The fundamental frequency difference of the laser, $f_{\text {rest }}$, was measured by a photo detector (PD), PD0, as the beat frequency of the two frequencies. Two beat frequencies, $f_{\text {beat } 1}$ and $f_{\text {beat } 2 \text {, between }}$ the fundamental frequency and frequencies modulated by Doppler effects at the moving part and at the sensing point of the transducer were measured by PD1 and PD2.

The force measured by the transducer, $F_{\text {trans, was }}$ calculated using the output signal of the transducer $V_{\text {trans }}$ that was stored in a dynamic strain recorder (DSR; model: DC-204R, Tokyo Sokki Kenkyujo) with its static calibration result. $F_{\text {trans }}$ is compared with the force measured as the inertial force of the mass, $F_{\text {mass }}$.

$F_{\text {mass }}$ is calculated by the following formulas.

$$
\begin{aligned}
& f_{\text {Doppler } 1}=-\left(f_{\text {beat } 1}-f_{\text {rest }}\right), \\
& v_{1}=\lambda_{\text {air }} f_{\text {Doppler } 1} / 2, \\
& a_{1}=d v_{1} / d t .
\end{aligned}
$$

The acceleration of contact point a 2 is calculated by a similar way,

$$
\begin{aligned}
& f_{\text {Doppler } 2}=-\left(f_{\text {beat } 2}-f_{\text {rest }}\right), \\
& v_{2}=\lambda_{\text {air }} f_{\text {Doppler } 2} / 2, \\
& a_{2}=d v_{2} / d t .
\end{aligned}
$$

To perform simultaneous measurements, three frequency counters and the DSR were triggered by a single signal that originated from a light switch composed of a laser diode (LD) and a PD.

\section{Results and Discussion}

Figure 2-5 shows the results of an impact test. Fig. 2 shows the force measured by the force transducer $F_{\text {trans }}$, the force acting on the moving part $F_{\text {mass}}$, and their difference $F_{\text {diff }}$, calculated by

$$
F_{\text {diff }}=F_{\text {trans }}-F_{\text {mass }} \text {. }
$$

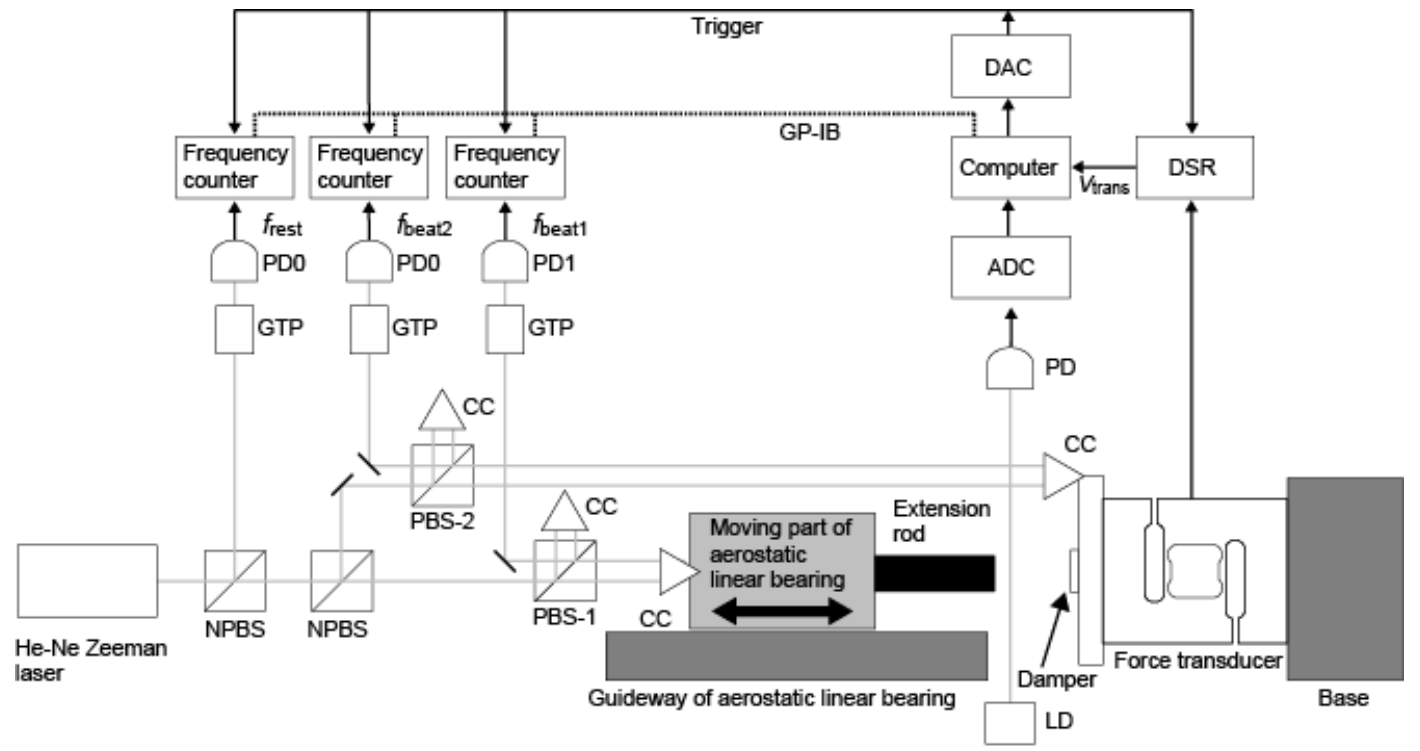

Fig. 1. Experimental setup. Code: $\mathrm{LD}=$ Laser diode, $\mathrm{PD}=$ Photo detector, $\mathrm{CC}=\mathrm{Corner}$ cube prism, $\mathrm{PBS}=\mathrm{Polarizing}$ beam splitter, NPBS = Non-polarizing beam splitter, GTP = Glan-Thompson prism, ADC = Analog-to-digital converter, $\mathrm{DAC}=$ Digital-to-analog converter, and DSR = Dynamic strain recorder 
$F_{\text {trans }}$ shows the electric response of the force transducer under the impact load. The difference between $F_{\text {trans }}$ and $F_{\text {mass }}$ is derived mainly from the difference between the static and the dynamic characteristics of the transducer. The root mean square (RMS) value of the difference between $F_{\text {trans }}$ and $F_{\text {mass }}$ during the collision, $0 \mathrm{~ms}$ $<t<14 \mathrm{~ms}$, was approximately $6.2 \mathrm{~N}$.

Figure 3 shows the relationship between the acceleration of the sensing part of the transducer $a_{2}$ and $F_{\text {diff- }}$. The plot shows a linear relationship between $a_{2}$ and $F_{\text {diff. }}$ The solid line in Fig. 3 shows a regression line with the following equation: $F_{\text {reg }}=-0.28 \times a_{2}$.

As shown in Fig. 3, the difference between the force measured by using the LMM and the force measured by the transducer is linear to the acceleration of the sensing part of the transducer because the dynamic error was caused by the inertial force of the transducer itself. Therefore, if we can

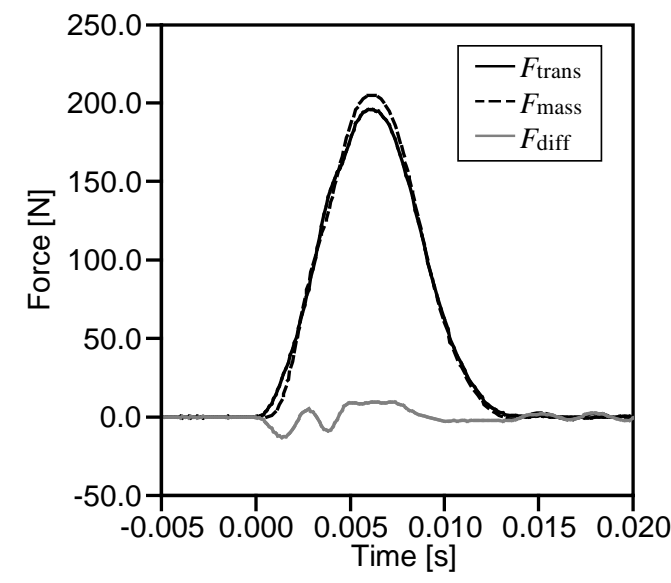

Fig. 2. Force measured with the transducer $F_{\text {trans }}$ and the force measured with the interferometer $F_{\text {mass }}$, and the difference between the two forces, $F_{\text {diff. }}$.

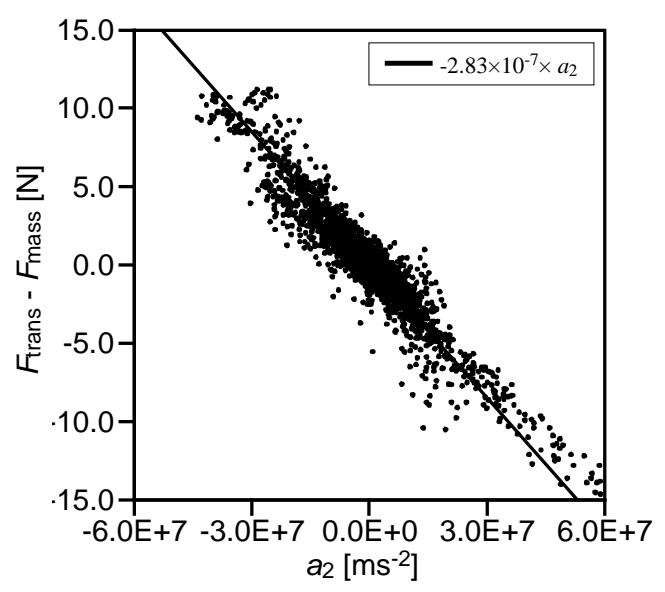

Fig. 3. Relationship between the acceleration of the sensing point of the transducer and $F_{\text {diff. }}$. get the acceleration of the transducer, we can correct the dynamic error. According to the principle of the strain gauge transducer, the transducer measures displacement of the sensing point based on the Hooke's law. Accordingly, the second time derivative of the output of the transducer should have linear relationship with the acceleration, farther more, with the dynamic error, as following equation:

$$
F_{\text {diff }}=C \times d^{2} F_{\text {trans }} / d t^{2},
$$

where $C$ is a parameter for dynamic error estimation. The parameter $C$ is obtained from the slope of the regression line determined by a least-square method, which resulted in $C=-2.49 \times 10^{-7} \mathrm{~s}^{2}$ in this experiment.

Figure 4 shows the difference between $F_{\text {trans }}$ and $F_{\text {mass }}$ and the inertial forces of the transducer that is estimated using the parameter $C, F_{\text {reg. }}$. The measurement error, $F_{\text {diff }}$, is almost the same as the result of the regression analysis, $F_{\text {reg }}$. From this result, the corrected force can be calculated using

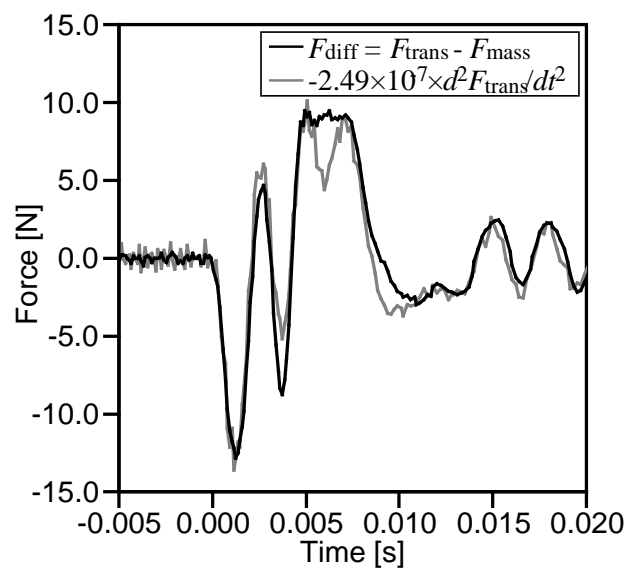

Fig. 4. Responses of $F_{\text {diff }}$ and $F_{\text {reg. }}$.

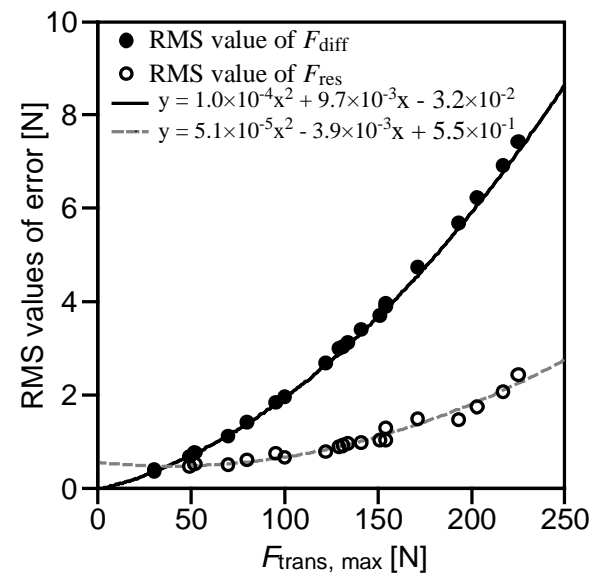

Fig. 5. RMS values of $F_{\text {diff }}=F_{\text {trans }}-F_{\text {mass }}$ and $F_{\text {res }}=F_{\text {corrected }}-F_{\text {mass }}$. 
the following equation:

$$
\begin{aligned}
F_{\text {correct }} & =F_{\text {trans }}-F_{\text {reg }} \\
& =F_{\text {trans }}-C \times d^{2} F_{\text {trans }} / d t^{2} \\
& =F_{\text {trans }}+2.49 \times 10^{-7} \times d^{2} F_{\text {trans }} / d t^{2} .
\end{aligned}
$$

Figure 5 shows the RMS values of the dynamic errors $F_{\text {diff, }}$ and the residual errors after dynamic correction $F_{\text {res, }}$,

$$
F_{\text {res }}=F_{\text {correct }}-F_{\text {mass }} \text {. }
$$

The RMS values of $F_{\text {diff }}$ and $F_{\text {res }}$ appear to be almost square functions of the maximum force $F_{\text {trans, max }}$ In Fig. 5, the solid and dashed curves represent the regression curves of the RMS values of $F_{\text {diff }}$ and $F_{\text {res }}$, respectively. The RMS values of the error between $F_{\text {diff }}$ and its regression value and that of the error between $F_{\text {res }}$ and its regression value are $1.34 \times 10^{-1} \mathrm{~N}$ and $3.58 \times 10^{-1} \mathrm{~N}$, respectively. These results show the potential for estimating the RMS values of the $F_{\text {diff }}$ and $F_{\text {res }}$ from the $F_{\text {trans }}$, max.

\section{Conclusions}

The method for measuring dynamic force applied to a force transducer and the method for correcting the difference between the applied force and the force measured by the transducer were reviewed. The dynamic force was measured by use of the LMM and the dynamic error was calculated. According to the relationship between the dynamic error and the output of the transducer, it is shown that the dynamic error can be corrected by the output of the transducer itself. Finally, the relationship between the impact force and the RMS value of the error were measured and the possibility of the estimation of the RMS errors from the peak force was shown. In the next step, we will attempt to investigate the parameter for dynamic correction for other types and other models of force transducers.

\section{Acknowledgment}

This work was supported in part by a research-aid fund of the Asahi Glass Foundation, a research-aid fund of the NSK Foundation for the Advancement of Mechatronics (NSK-FAM) and the Grant-in-Aid for Scientific Research (B) 24360156 (KAKENHI 24360156)..

\section{References}

(1) R. Kumme : "Investigation of the comparison method for the dynamic calibration of force transducers",
Measurement, Vol. 23, pp.239-245, 1998

(2) Y.-K. Park, R. Kumme, and D.-Im Kang : "Dynamic investigation of a binocular six-component force-moment sensor", Meas. Sci. Technol., Vol. 13, p. 1311, 2009

(3) Y. Fujii : "Toward establishing dynamic calibration method for force transducers", IEEE Trans. Instrum. Meas., Vol. 58, pp. 2358-2364, 2009

(4) Y. Fujii : "Measurement of the electrical and mechanical responses of a force transducer against impact forces", Rev. Sci. Instrum., Vol. 77, Number, 085108, 2006

(5) Y. Fujii : "Method for correcting the effect of the inertial mass on dynamic force measurements", Meas. Sci. Technol., Vol. 18, No. 5, pp. N13-N20, 2007

(6) Y. Fujii and K. Maru : "Self-correction method for dynamic measurement error of force sensors", Exp. Tech., Vol. 35, No. 3, pp. 15-20, 2011

(7) Y. Fujii : "Frictional characteristics of an aerostatic linear bearing", Tribol. Int., Vol. 39, No. 9, pp. 888-896, 2006 